\title{
The relationship between smartphone addiction and musculoskeletal pain prevalence among young population: a cross-sectional study
}

\author{
Rustem Mustafaoglu ${ }^{1}$, Zeynal Yasaci ${ }^{2,3}$, Emrah Zirek $^{2,4}$, Mark D. Griffiths ${ }^{5}$, and Arzu Razak Ozdincler ${ }^{6}$ \\ 'Department of Physiotherapy and Rehabilitation, Division of Physiotherapy and Rehabilitation, Faculty of Health Sciences, Istanbul University- \\ Cerrahpasa, Istanbul, Turkey \\ ${ }^{2}$ Department of Physiotherapy and Rehabilitation, Institute of Graduate Studies, Istanbul University-Cerrahpasa, Istanbul, Turkey \\ ${ }^{3}$ Division of Physiotherapy and Rehabilitation, Faculty of Health Sciences, Harran University, Sanliurfa, Turkey \\ ${ }^{4}$ Division of Physiotherapy and Rehabilitation, Faculty of Health Sciences, Bingol University, Bingol, Turkey \\ 5International Gaming Research Unit, Psychology Department, Nottingham Trent University, Nottingham, UK \\ ${ }^{6}$ Division of Physiotherapy and Rehabilitation, Faculty of Health Sciences, Biruni University, Istanbul, Turkey
}

Received June 1, 2020

Revised September 18, 2020

Accepted September 21, 2020

Handling Editor: Kyung-Joon Lim

\section{Correspondence}

Rustem Mustafaoglu

Department of Physiotherapy and Rehabilitation, Division of Physiotherapy and Rehabilitation, Faculty of Health Sciences, Istanbul University-Cerrahpasa, Büyükçekmece Yerleşkesi Alkent 2000 Mah. Yiğittürk Cad. No:5/9/1 Büyükçekmece, Istanbul, Turkey Tel: +90-212-414-15-00 Fax: +90-212-866-38-31 E-mail: ahiska_1944@hotmail.com
Background: In the literature, there have been debates as to whether smartphone use has negative effects on physical and mental health. The present study investigated the extent to which smartphone addiction impacts on musculoskeletal pain prevalence among university students.

Methods: The questionnaire consisted of three sections: demographic information, the Smartphone Addiction Scale (SAS), and the modified Nordic Musculoskeletal Questionnaire.

Results: A total of 249 participants were included in this cross-sectional study. The body parts that were reported with highest prevalence of musculoskeletal pain were the upper back (70.3\%), neck (65.9\%), and wrists/hands (68.7\%). The SAS scores were correlated with duration of smartphone use on a typical day $(P=0.001)$, duration of owning a smartphone $(P=0.027)$, and musculoskeletal pain prevalence in the neck $(P=0.001)$, wrists/hands $(P=0.001)$, shoulders $(P=0.025)$, and upper back $(P=0.023)$. The SAS score was significantly associated with prevalence of musculoskeletal pain in the neck (odd ratio [OR], 1.08; 95\% confidence interval [Cl], $0.98-1.10 ; P=0.002)$, wrists/hands (OR, 1.07; 95\% Cl, 0.97-1.09; $P=0.001)$, and upper back (OR, 1.10; $95 \% \mathrm{Cl}, 0.98-1.11 ; P=0.033$ ).

Conclusions: The findings indicated that the upper back, neck, and wrists/hands have a higher prevalence of musculoskeletal pain among smartphone users, particularly those with a smartphone addiction. Smartphone addiction scores were correlated with duration of smartphone use on a typical day, duration of owning smartphone, and musculoskeletal pain prevalence in the neck, wrists/hands, shoulders, and upper back.

Key Words: Behavior, Addictive; Chronic Pain; Cross-Sectional Studies; Mental Health; Musculoskeletal Pain; Prevalence; Smartphone; Surveys and Questionnaires. (c) This is an open-access article distributed under the terms of the Creative Commons Attribution Non-Commercial License (http://creativecommons.org/licenses/by-nc/4.0/), which permits unrestricted non-commercial use, distribution, and reproduction in any medium, provided the original work is properly cited.

(C) The Korean Pain Society, 2021
Author contributions: Rustem Mustafaoglu: Writing/manuscript preparation; Zeynal Yasaci: Investigation; Emrah Zirek: Investigation; Mark D. Griffiths: Writing/manuscript preparation; Arzu Razak Ozdincler: Writing/ manuscript preparation. 


\section{INTRODUCTION}

Smartphones offer a range of mobile applications for communication, education, and entertainment and have become an essential part of everyday life. The smartphone is one of the most popular technological devices among many ages worldwide. A recent study showed that $79 \%$ of the population between the age 18-44 years have their smartphones with them almost all the time, with only two hours of their waking day spent without their smartphone in hand [1]. Innovations in smartphone models, including surfing the internet, social media apps, gaming apps, portable media players, compact digital cameras, and highresolution touchscreens, have been a factor in the frequent use of and "addiction" to smartphones [2]. The number of smartphone users worldwide was estimated to be 3.5 billion at the end of 2019 and is expected to reach 3.8 billion by 2021 [3].

Addiction to smartphones can cause serious problems, especially for students. These problems, which now appear to start in childhood, can manifest as a social problem for a minority of individuals over time. These addictions have been identified as non-chemical behavioural addictions involving human-machine interaction, or socalled "technological addictions" [4]. "Gambling disorder" and "Internet gaming disorder" are currently the only non-substance-related disorders defined as behavioral addictions in the fifth edition of the Diagnostic and Statistical Manual of Mental Disorders (DSM-5) [5]. Smartphone addiction has a some similarities to DSM- 5 substancerelated disorders [6]. Consequently, in recent years, a number of psychometric scales have been developed to assess problematic smartphone use and/or smartphone addiction $[2,6,7]$.

Because smartphones are now being used by individuals more frequently than computers every day, it is not surprising that various negative effects of excessive smartphone use have emerged. In daily life, smartphone users browse the internet, use social media, chat with other users, play games, gamble, listen to music, and perform many other activities on their smartphones $[8,9]$. During these tasks, individual may be engaged in one position for a long time without moving or making specific movements repeatedly leading to various musculoskeletal disorders [10-12]. Most smartphone tasks require users to stare sharply downwards or to hold their arms out in front of them to read the screen which makes their head move forward and can cause an excessive anterior curve in the lower cervical vertebrae and an excessive posterior curve in the upper thoracic vertebrae to maintain balance, which places stress on the cervical spine and the neck muscles [13]. Forward head posture is one of the most commonly recognized poor postures in the sagittal plane. Incorrect posture of the head and neck has been correlated with chronic musculoskeletal pain $[14,15]$.

The incidence of musculoskeletal disorders of hand, wrist, forearm, arm, and neck has been increasing worldwide due to the prolonged, forceful, low amplitude, repetitive use of hand held devices. Continuous repetitive movements with the thumb and fingers have been identified as risk factors which may lead to disorders of the thumb and its musculature such as tendinosis of the extensor pollicis longus, or myofacial pain syndrome in the hand. In the literature, several studies have examined the impact of smartphone usage on the musculoskeletal system [14,1619]. A retrospective study found that all the hand-held device user participants reported pain in the thumb and forearm with associated burning, numbness, and tingling around the thenar eminence of the hand [16]. Similar results were found among adolescents who use electronic devices where musculoskeletal pain $(61 \%)$ and musculoskeletal pain syndrome (33\%) were reported.

Although numerous studies conducted on smartphone addiction are generally focused on mental health (stress, academic performance, satisfaction with life, loneliness, depression, anxiety, etc.) [20-22], few studies have examined the musculoskeletal risks associated with problematic smartphone use. It is necessary to evaluate the physical changes that occur during the use of smartphones, especially during the repeated movements in the smartphone use, and to determine whether their use is a risk factor that can induce musculoskeletal disorders. To date, no comprehensive study (i.e., studies evaluating musculoskeletal problems using standardized assessment tools) has directly focused on the adverse musculoskeletal health effects of problematic smartphone use. Therefore, the aim of this study was to investigate smartphone addiction level and its impact on musculoskeletal pain prevalance among university students.

\section{MATERIALS AND METHODS}

\section{Participants and settings}

The present study used convenience sampling. To be included in the study, participants had to be aged between 18-25 years with a minimum of 25 text messages or emails per day, browsing the Internet and/or playing games for more than one hour a day using their smartphone. The exclusion criteria were any participants with neck, shoulder, upper back, lower back, elbow, or wrist-hand musculoskeletal trauma, and those with congenital deformities, serious surgical or neurological diseases, limb injuries, 
or limb pain in the prior six months. Before completing the survey, the purpose of the study was explained to the participants, and participants were assured that their data were anonymous and confidential. All procedures were performed in accordance with the Declaration of Helsinki, and ethical approval for this study was obtained from the Human Research Ethics Committee of Bakirkoy Dr. Sadi Konuk Training and Research Hospital (IRB: 2018-03-17).

\section{Sample size}

The sample size was calculated using $G^{*}$ Power 3.1 software (Heinrich-Heine-Universität Düsseldorf, Düsseldorf, Germany). The lowest sample size required for the present study was 232, which was estimated to enable the detection of an odds ratio (OR) of 1.6 with $80 \%$ power and an alpha probability of 0.05 . This expected sample size was based on the OR reported by a longitudinal populationbased cohort study with young adults on the risk of neck/ upper back pain with texting on mobile phones [23]. With an expected drop-out rate of $30 \%$ due to possible nonresponses or other reasons, a total sample of 300 students was initially planned to be included in the present study. Due to absence during the study period, incomplete questionnaires, or lack of interest in the study, a total of 249 university students' data remained for analysis.

\section{Data collection instruments}

\section{1) Demographics}

Age, sex, weight, height and body mass index (BMI), daily smartphone usage time, duration of owning a smartphone, and the purposes of smartphone use in a typical day, including: (1) talking (voice-to-voice), (2) text messaging, (3) listening to music, (4) watching videos, (5) social networking, (6) gaming, and (7) other.

\section{2) Smartphone Addiction Scale (SAS)}

The SAS is a 33-item scale in which responses to items are given on a six-point Likert-type scale from 1 (strongly disagree) to 6 (strongly agree) [2] . It is based on the Internet Addiction Test [24] and comprises six factors: daily-life disturbance, positive anticipation, withdrawal, cyberspaceoriented relationships, overuse, and tolerance. Higher scores indicate a higher risk of smartphone addiction. The total scores ranged from 33 to 198. A cut-off point for smartphone addiction was not reported in the original scale, with higher scores indicating a greater risk of being addicted to smartphones. A reliability and validity study of the Turkish version of the SAS was conducted by Demirci et al. [25], and the Cronbach's alpha was 0.94 .

3) Modified Nordic Musculoskeletal Questionnaire (mNMQ)

In the present study, the mNMQ was used [26]. The mNMQ consists items relating to six body regions (neck, shoulders, upper back, lower back, elbow, and wrists-hands) from the original NMQ, and assesses upper body musculoskeletal symptoms related to any pain or numbness in the previous 12 months and last seven days. The mNMQ investigates pain and numbness in the body regions that are used most frequently during smartphone use, enabling participants to answer "yes" or "no" by using a simple body image. The participants were asked whether the pain that had developed with in the past year prevented them from doing normal work (at home or outside the home) or whether hospitalization was required due to this pain. The Turkish version of NMQ was used in the present study [27].

\section{Statistical analysis}

All statistical analyses were performed using SPSS version 22.0 (IBM Co., Armonk, NY). The one-sample KolmogorovSmirnov test was performed to check the normality of distribution of each continuous variable. Data are presented as mean \pm standard deviation (SD), and frequencies. Because the data were normally distributed, Pearson's correlation tests were performed to assess the bivariate correlation between two continuous variables, and pointbiserial correlations were used to test the correlation between one continuous and one dichotomous variable. In addition, an independent sample t-test was performed to establish whether there were any significant differences in mean SAS scores in relation to each type of body part pain experienced by participants. Logistic regression models were constructed to predict the body parts experiencing discomfort. Each mMNQ assessed musculoskeletal body part discomfort by using pain versus indolence as a dependent variable in the logistic regression model. Sex, duration of smartphone use on a typical day, duration of owning a smartphone, and SAS scores were considered as independent variables. The ORs and $95 \%$ confidence intervals (CIs) were reported. Statistical significance was based on $P$-values $<0.05$.

\section{RESULTS}

\section{Descriptive statistics}

A total of 249 participants completed the questionnaire 
( $67.5 \%$ females). The response rate was $83.0 \%$. A total of 51 individuals were excluded from the study because they withdrew from the study and/or had missing data. Participants' mean age was $21.3 \pm 1.9$ (range 18-25) years and mean BMI was $22.1 \pm 3.2$ (range $17.1-40.8$ ) $\mathrm{kg} / \mathrm{m}^{2}$. It was also found that the most common uses by participants for their smartphone were making phone calls (98.4\%) and social networking (96.0\%). In addition, the average SAS score was $77.5 \pm 23.9$ (range 33-175). Table 1 presents general

Table 1. General characteristics of the participants and smartphone use attitude

\begin{tabular}{lc}
\hline \multicolumn{1}{c}{ Characteristic } & Value \\
\hline Sex & \\
Female & $168(67.5)$ \\
Male & $81(32.5)$ \\
Age (yr) & $21.3 \pm 1.9$ \\
Weight (kg) & $63.1 \pm 12.3$ \\
Height (cm) & $168.5 \pm 9.1$ \\
Body mass index (kg/m $\left.{ }^{2}\right)$ & $22.1 \pm 3.2$ \\
Purposes of smartphone use & \\
Making phone calls & $245(98.4)$ \\
Text messaging & $220(88.4)$ \\
Gaming & $142(57.0)$ \\
Listening to music & $209(83.9)$ \\
Watching videos & $177(71.1)$ \\
Social networking & $239(96.0)$ \\
Other & $97(39.0)$ \\
Duration of smartphone use on a typical day (hr) & \\
1-2 & $53(21.3)$ \\
3-4 & $104(41.8)$ \\
$5-6$ & $59(23.7)$ \\
$>$ > 6 & $33(13.3)$ \\
Duration of owning smartphone (yr) & \\
1-3 & \\
$4-6$ & $20(8.0)$ \\
$7-9$ & $45(18.1)$ \\
SAS score 9 & $77.5 \pm 23.9$ \\
\hline
\end{tabular}

Values are presented as mean \pm standard deviation or number (\%). SAS: Smartphone Addiction Scale. characteristics of the participants and their smartphone use attitude.

\section{Musculoskeletal pain prevalence}

Prevalence of musculoskeletal pain during past year and past week of the participants is shown in Table 2. Among the one-year musculoskeletal pain symptoms, upper back (70.3\%), neck (65.9\%), wrists/hands (68.7\%), and shoulders (56.6\%) were the body parts with the highest prevalence of pain. Similarly, among the past-week musculoskeletal pain symptoms, wrists/hands (58.2\%), upper back (51.0\%), and neck (45.4\%) were the body parts with the highest prevalence of pain. Participants who experienced pain in the upper back (22.5\%), lower back (18.5\%), wrists/hands (18.5\%), and neck (16.9\%) reported that they were unable to perform routine work at home or their workplaces for a day or more. Apart from elbow $(P=0.104)$ and lower back pain $(P=0.221)$, SAS scores of individuals with pain in all other areas were significantly higher than those without pain (all $P$ values $<0.05$; see Table 3 ).

\section{The correlations of SAS scores with participants' characteristics, smartphone use, and musculoskeletal pain prevalence}

Correlation coefficients for the study variables are presented in Table 4 and Fig. 1. The SAS scores were positively correlated with duration of smartphone use on a typical day $(\mathrm{r}=0.681 ; P=0.001)$, duration of owning a smartphone ( $\mathrm{r}=0.301 ; P=0.027$ ), musculoskeletal pain prevalence in the neck $(\mathrm{r}=0.457 ; P=0.001)$, shoulder $(\mathrm{r}=0.145 ; P=0.025)$, wrists/hands ( $\mathrm{r}=0.504 ; P=0.001)$, and upper back $(\mathrm{r}=$ $0.148 ; P=0.023)$. The findings demonstrated that smartphone addiction was associated with high musculoskeletal pain prevalence in the neck, wrists/hands, shoulders, and upper back.

Table 2. Prevalence of musculoskeletal pain during past year and past week in participants by modified Nordic Musculoskeletal Questionnaire

\begin{tabular}{lccc}
\hline Body part & Past-year musculoskeletal pain & Past-week musculoskeletal pain & $\begin{array}{c}\text { Prevented you from doing your normal work } \\
\text { (at home or away from home) during the past year }\end{array}$ \\
\hline Neck & $164(65.9)$ & $113(45.4)$ & $42(16.9)$ \\
Shoulder & $141(56.6)$ & $107(43.0)$ & $37(14.9)$ \\
Elbow & $39(15.7)$ & $29(11.6)$ & $14(5.6)$ \\
Wrists/hands & $171(68.7)$ & $145(58.2)$ & $46(18.5)$ \\
Upper back & $175(70.3)$ & $127(51.0)$ & $56(22.5)$ \\
Lower back & $111(44.6)$ & $79(31.7)$ & $46(18.5)$ \\
\hline
\end{tabular}

Values are presented as number (\%). 


\section{Factors associated with musculoskeletal pain in at least one body site at any time}

The logistic regression analysis indicated that past year

Table 3. Comparison of SAS scores and presence or absence of pain in six body parts

\begin{tabular}{lcccc}
\hline \multicolumn{1}{c}{ Body part } & Pain & SAS score & t-score & $P$ value $^{\mathrm{a}}$ \\
\hline Shoulder & $(+)$ & $80.3 \pm 25.9$ & -2.255 & 0.025 \\
& $(-)$ & $73.2 \pm 19.7$ & & \\
Elbow & $(+)$ & $76.4 \pm 30.1$ & 1.634 & 0.104 \\
& $(-)$ & $73.2 \pm 21.8$ & & \\
Wrists/hands & $(+)$ & $88.5 \pm 24.3$ & -8.981 & 0.001 \\
& $(-)$ & $64.4 \pm 15.2$ & & \\
Neck & $(+)$ & $85.6 \pm 22.8$ & -7.905 & 0.001 \\
& $(-)$ & $62.9 \pm 18.1$ & & \\
Upper back & $(+)$ & $83.5 \pm 23.4$ & -6.391 & 0.001 \\
& $(-)$ & $64.3 \pm 19.1$ & & \\
Lower back & $(+)$ & $73.9 \pm 24.2$ & 1.123 & 0.221 \\
& $(-)$ & $72.1 \pm 22.1$ & & \\
\hline
\end{tabular}

Values are presented as mean \pm standard deviation.

SAS: Smartphone Addiction Scale, (+): yes, (-): no.

andependent sample $t$-test.
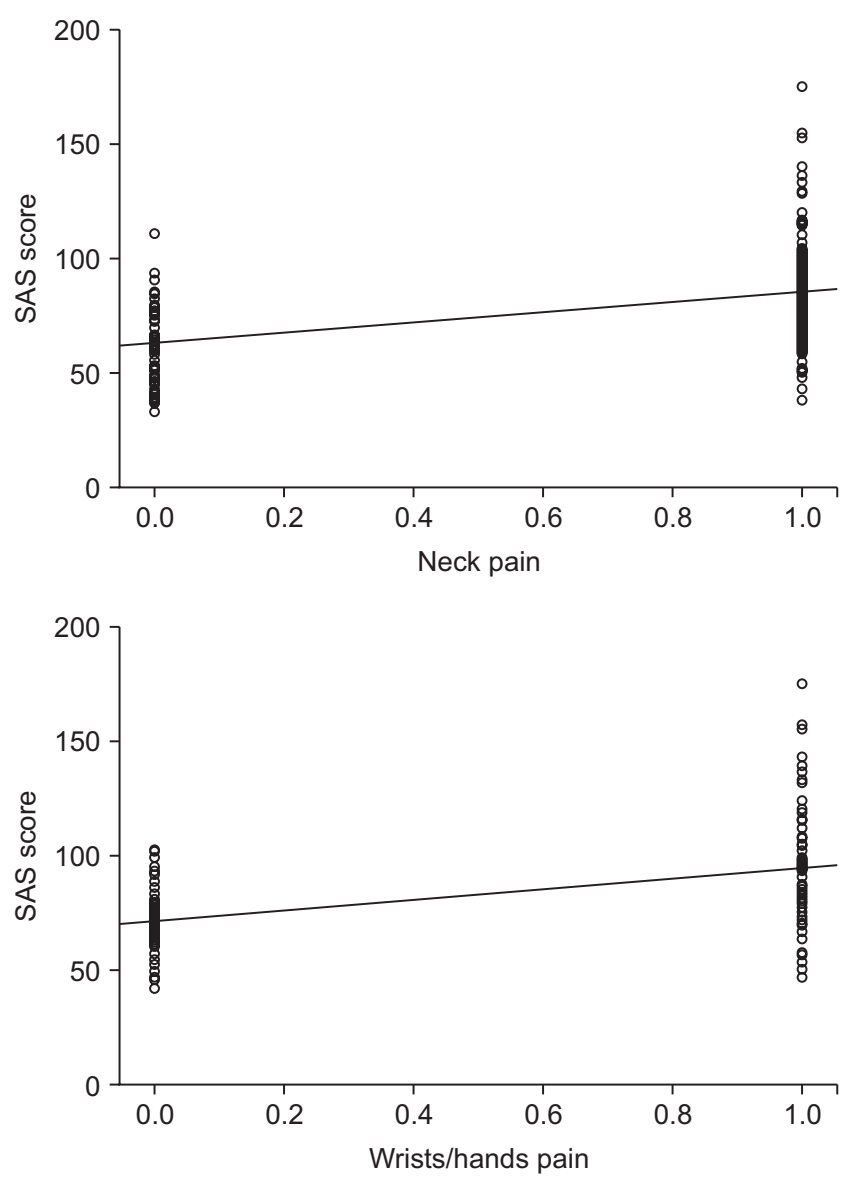

musculoskeletal pain prevalence in the neck (OR, 1.78; 95\% CI, 0.98-3.23; $P=0.041$ ), shoulders (OR, 2.59; 95\% CI, $1.43-4.70 ; P=0.002)$, wrists/hands (OR, 2.15; 95\% CI, 1.15-

Table 4. The correlations between SAS scores and participants' characteristics, smartphone usage, and musculoskeletal pain prevalence of the body parts

\begin{tabular}{lcc}
\hline \multirow{2}{*}{ Characteristic } & \multicolumn{2}{c}{ SAS score } \\
\cline { 2 - 3 } & r or r $_{\text {bp }}$ & $P$ value \\
\hline Age & 0.046 & $0.932^{\mathrm{a}}$ \\
Sex & 0.041 & $0.529^{\mathrm{b}}$ \\
Duration of smartphone use on a typical day & 0.681 & $0.001^{\mathrm{a}}$ \\
Duration of owning smartphone & 0.301 & $0.027^{\mathrm{a}}$ \\
Neck & 0.457 & $0.001^{\mathrm{b}}$ \\
Shoulder & 0.145 & $0.025^{\mathrm{b}}$ \\
Elbow & 0.108 & $0.104^{\mathrm{b}}$ \\
Wrists/hands & 0.504 & $0.001^{\mathrm{b}}$ \\
Upper back & 0.148 & $0.023^{\mathrm{b}}$ \\
Lower back & 0.107 & $0.099^{\mathrm{b}}$ \\
\hline
\end{tabular}

SAS: Smartphone Addiction Scale.

aPearson's correlation coefficient test was used. $r=$ correlation coefficient. ${ }^{b}$ Point-biserial correlation test was used. $r_{b p}=$ point-biserial correlation coefficient.
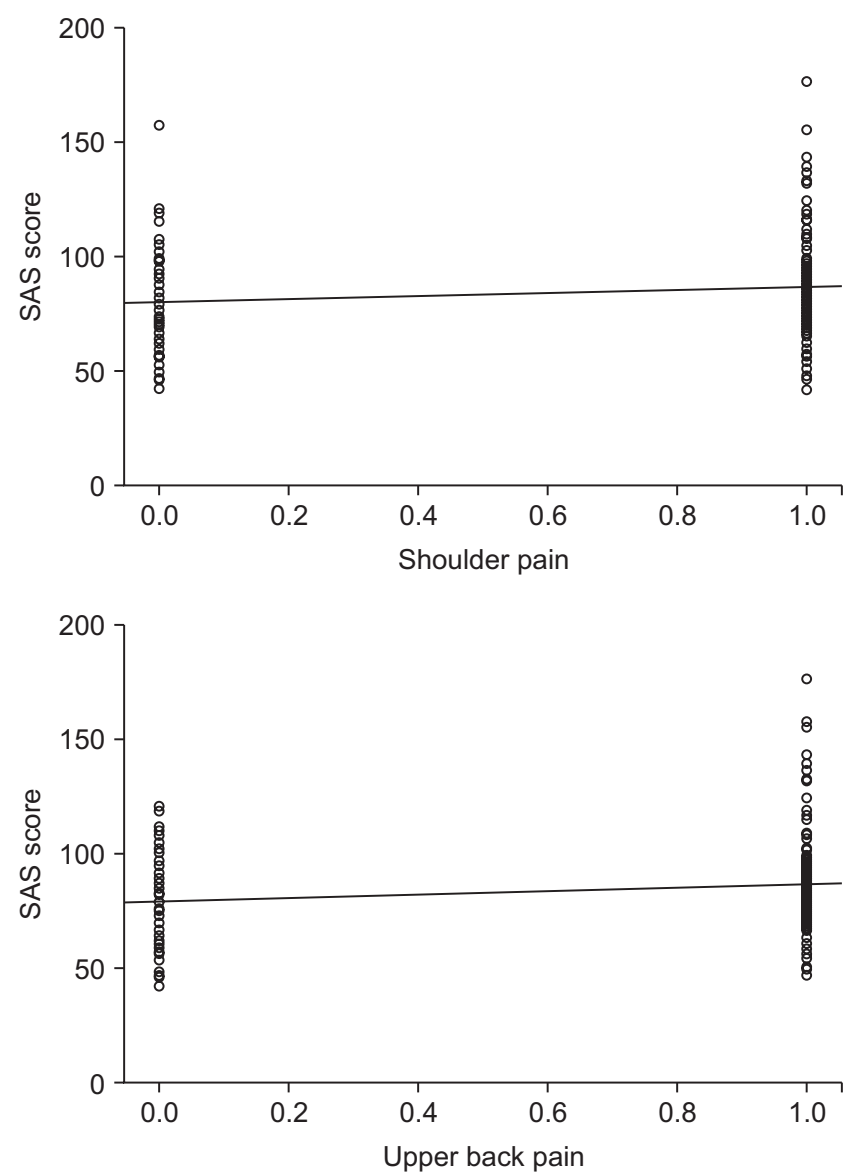

Fig. 1. Correlations between the Smartphone Addiction Scale (SAS) and presence of musculoskeletal pain in the body parts. 
3.90; $P=0.010)$, and upper back (OR, 2.83; 95\% CI, 1.545.19; $P=0.001$ ) was significantly less among males than females. Smartphone use of over six hours in a typical day was significantly associated with high musculoskeletal pain prevalence in the neck (OR, 1.68; 95\% CI, 0.46-4.10; $P$ $=0.031$ ), shoulders (OR, 2.12; 95\% CI, 0.68-5.88; $P=0.020)$, and wrists/hands (OR, 2.55; 95\% CI, 0.53-7.51; $P=0.010)$ in comparison to the reference group (1-2 hr daily smartphone use). The subgroup which had owned a smartphone for over nine years was significantly associated musculoskeletal pain prevalence in the neck (OR, 3.94; 95\% CI, 0.98-8.23; $P=0.001)$ and wrists/hands (OR, 5.55; 95\% CI, 0.84-12.85; $P=0.001$ ) compared to the reference group (1-3 yr owning a smartphone). SAS score was significantly associated with musculoskeletal pain prevalence in the neck (OR, 1.08; 95\% CI, 0.98-1.10; $P=0.002$ ), wrists/hands (OR, 1.07; 95\% CI, 0.97-1.09; $P=0.001)$, and upper back (OR, 1.10; 95\% CI, 0.98-1.11; $P=0.033$ ) (Table 5).

\section{DISCUSSION}

According to the results of the present study, musculoskeletal pain symptoms in the past year and past week showed higher pain prevalence in the upper back, neck, and wrist/ hands. Participants experienced pain in the upper back, waist, and wrists/hands, where they were unable to perform routine work in their homes or workplaces for a day or more. Smartphone addiction was associated with (1) duration of smartphone use on a typical day, (2) duration of owning a smartphone, and (3) musculoskeletal pain prevalence in the neck, wrists/hands, shoulders, and upper back. The logistic regression analysis showed that spending over six hours a day on a smartphone was significantly associated with high musculoskeletal pain prevalence in the neck, shoulders, and wrists/hands. Moreover, owning a smartphone for over nine years was also significantly associated with high musculoskeletal pain prevalence in the neck and wrists/hands. Smartphone addiction was significantly associated with musculoskeletal pain prevalence in the neck, wrists/hands, and upper back.

In the literature, a study in South Korea by Kwon et al. [2] reported SAS scores of 104.5 points for males and 112.7 points for females, and 110.02 for both groups combined, and there was no sex difference in SAS scores. On the other hand, in Turkey, Demirci et al. [28] reported that the mean SAS score was 75.68. In the present study, it was found that the SAS mean score was 77.5 , and SAS scores were correlated with duration of smartphone use on a typical day, duration of owning a smartphone, and musculoskeletal pain prevalence in the neck, wrists/hands, shoulders, and upper back. The results also suggest that the most impor- tant indicators of smartphone addiction are the excessive use of the smartphone due to its ability to facilitate and host many different activities (online surfing, gaming, social networking, etc.). Although the results of the present study are similar to the results of Demirci et al. [28], the fact that SAS scores were lower than the results of Kwon et al. [2] is most probably due to cultural reasons.

The use of smartphones has grown rapidly over the past decade. This can lead to problemetic smartphone use for a minority of indivduals. Excessive use of a smartphones may cause various physical and psychological health problems for such individuals. In the literature, some studies have investigated the relationship between smartphone/mobile phone use and musculoskeletal symptoms and syndromes $[14,16,26,29]$. The findings showed that the upper back, neck, and wrists/hands were the most painreported body parts among the participants. Some participants experienced pain in the upper back, lower back, and wrists/hands, and were unable to perform routine work at home or workplaces for a day or more. The findings here echoed the research by Kim and Kim [17] who reported the most frequent musculoskeletal pain was in areas of the body related to smartphone use (i.e., shoulders and neck). The authors found that smartphone use was correlated with musculoskeletal pain. AlAbdulwahab et al. [14] reported that smartphone addiction can lead to a significant neck disability due to poor posture associated smartphone use. The authors suggested that individuals make efforts to reduce the time spent using smartphones and to maintain an appropriate posture while using them because prolonged smartphone use can lead to faulty postures such as a forward head posture. Sustaining this forward head posture can produce injuries to the cervical spine and cause cervical pain [30].

Pain of the cervical muscles is increased with prolonged smarthpone use, and this emphasizes the importance of long duration smartphone use as a risk factor in the development of ergonomic disorders. Consequently, it was suggested that correcting posture during use and break time for at least 20 minutes was required to avoid the negative effects of prolonged smartphone use [31]. According to the authors, pain in the musculoskeletal system can occur when smartphones are used continuously, without rest, and when poor posture persists for long periods of time. Repeated movements in a static position can lead to various problems, such as pain in the shoulder and neck. When using smartphones, the upper extremities and shoulders often perform repeated movements, while the upper back stabilizes the head and neck for best viewing. Yang et al. [26] showed that the (1) relationship between smartphone use and musculoskeletal symptoms was associated with the duration of the smartphone ancillary 


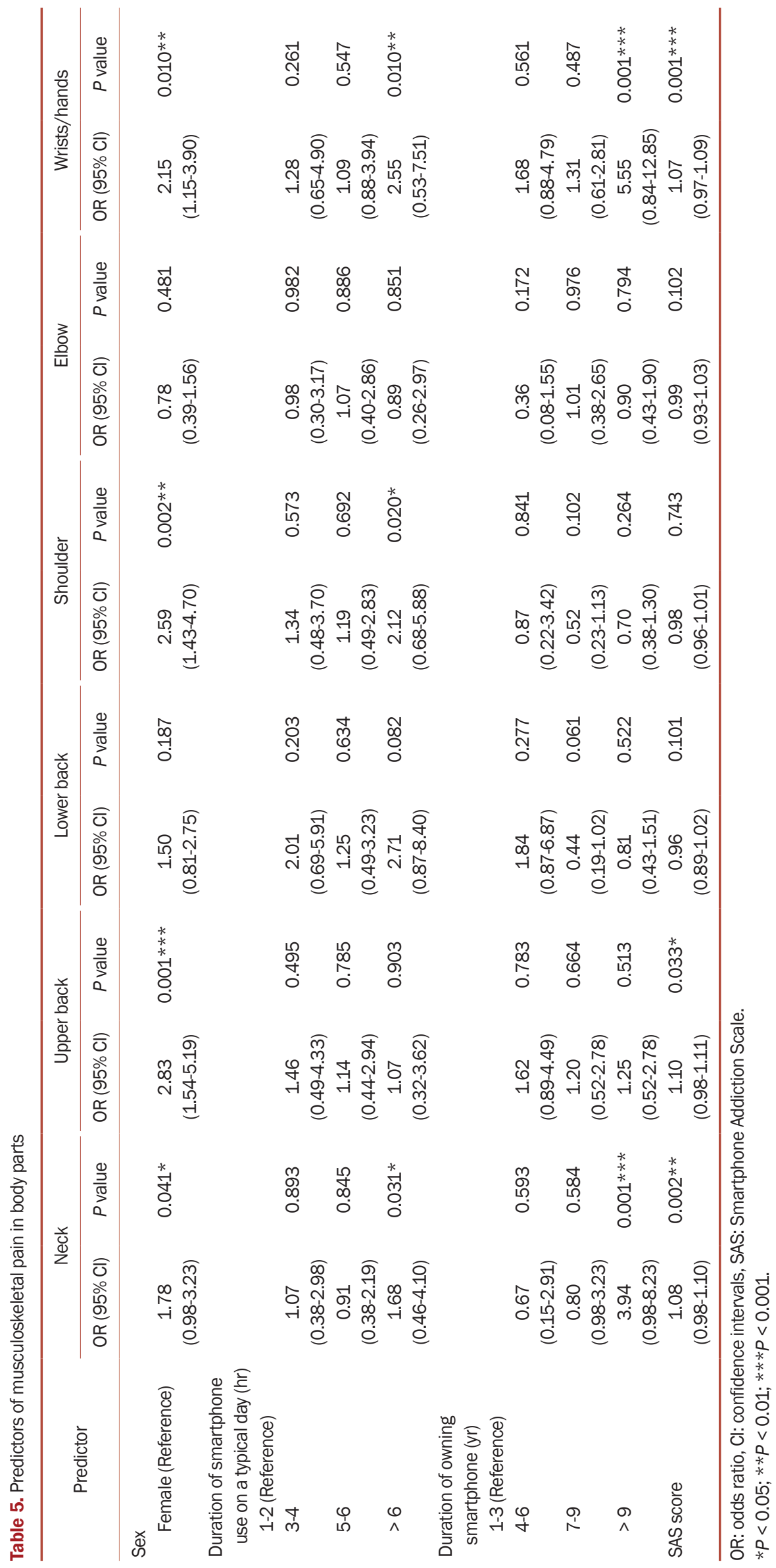


function use, and (2) hours spent talking on the phone was a predictor of upper back pain.

Hakala et al. [32] also reported that frequent use of mobile phones increased the risk of neck, shoulder, and lower back pain in adolescents. In a recent study, Toh et al. [33] reported that after adjusting for potential confounders, the number of hours a day spent on smartphones was associated with increased risk of neck/shoulder, upper back, arm, and wrist/hand discomfort. In another study, Hegazy et al. [29] reported (using an adjusted multiple logistic regression analysis) that excessive smartphone use was significantly associated with self-reported sleep disturbance, headache, fatigue, depression, nervousness, and musculoskeletal pain. Similarly, in our study, it was observed that high SAS scores, which are an indicator of excessive phone use, were associated with the presence of neck, shoulder, upper back, and wrist/hand pain symptoms. This is because, during phone use, sitting with the head bent forward, without supporting the arms, causes a static load on the neck and shoulder muscles, resulting in increased pain in the neck/upper back. In general, sustained muscle load and repetitive movements are considered to be risk factors for developing musculoskeletal disorders. Additionally, the present study found that past-year neck, shoulder, wrist/hand, and upper back musculoskeletal pain was significantly less in males than females. Similarly, Demirci et al. [28] reported that female sex and low age were independent predictors of excessive smartphone use and that female and young users may be vulnerable to excessive smartphone use or addiction. This may explain the higher prevalence among females than males in the present study, because there are differences in the purpose of smartphone use between males and females.

Studies have reported that females are more inclined to use smartphones for communication and social networking functions, which play prominent roles in their life, while males use smartphones to play games and watch videos, which is a more diversified type of usage [34]. Continuous muscle contractions in the neck and upper extremities can cause microscopic damage to the muscles during the performance of smartphone tasks. Neck disability among smartphone users may be associated with a frequent neck flexion posture that alters the natural curve of the cervical spine and increases the amount of stress on the cervical spine, causing irritation, spasm in the surrounding skeletal structures, and proprioception deficits in the cervical vertebra. Naturally, and as found in the present study, pain in these specific areas (neck, shoulders, and wrists/hands) has been associated with long-term smartphone use during the day and long-term smartphone ownership.

In the present study, there were some limitations. First, since the study was a cross-sectional study, cause-andeffect relationships cannot be determined, and the results are therefore somewhat limited. To confirm and expand on the results here, longitudinal or prospective experimental studies are needed. Secondly, all participants were university students (with a slight bias towards female students) and do not represent the total population of university students, therefore results cannot be generalized to other populations. Thirdly, the study did not assess and adjust for potential confounders (i.e., mental health and physical activity). Fourthly, all the data were self-reported and therefore were subject to well-known method biases. There are insufficient data to draw firm conclusions about the relationship between problematic smartphone usage and pain experience due to the various other possible factors, including biopsychosocial factors, that negatively effect the musculoskeletal system. Finally, the SAS does not provide any clinical diagnosis for smartphone addiction as it is a tool that was developed to provide an indication of those who might be at risk of smartphone addiction. This should be taken into account when interpreting any of the statistics related to SAS scores.

The results of the present study show that smartphone users experience musculoskeletal pain in their upper back, neck, and wrists/hands. High smartphone addiction scores were significantly correlated with duration of smartphone use on a typical day, duration of owning a smartphone, and musculoskeletal pain in the neck, wrists/ hands, shoulders, and upper back. The duration of smartphone use on a typical day is associated with increased risk of neck, shoulder, and wrist/hand musculoskeletal pain. In addition, long-term smartphone ownership is associated with increased risk of neck and wrist/hand musculoskeletal pain. Further study needs to be carried out on a larger cohort for more generalizable results, representing the whole population. Furthermore, attention should be directed towards the safe use of technology and increasing public awareness of the hazards of excessive and potentially problematic smartphone use.

\section{CONFLICT OF INTEREST}

No potential conflict of interest relevant to this article was reported.

\section{FUNDING}

No funding to declare. 


\section{ORCID}

Rustem Mustafaoglu, https://orcid.org/0000-0001-7030-0787

Zeynal Yasaci, https://orcid.org/0000-0001-9496-8095

Emrah Zirek, https://orcid.org/0000-0002-0546-5961

Mark D. Griffiths, https://orcid.org/0000-0001-8880-6524

Arzu Razak Ozdincler, https://orcid.org/0000-0003-1783-3992

\section{REFERENCES}

1. Neupane S, Ali UTI, Mathew A. Text neck syndrome-systematic review. Imp J Interdiscip Res 2017; 3: 141-8.

2. Kwon M, Lee JY, Won WY, Park JW, Min JA, Hahn C, et al. Development and validation of a smartphone addiction scale (SAS). PLoS One 2013; 8: e56936.

3. Statista. Number of smartphone users from 2016 to 2021 [Internet]. New York (NY): Statista; 2020. Available at: https:// www.statista.com/statistics/330695/number-of-smartphone-users-worldwide/.

4. Griffiths M. Gambling on the internet: a brief note. J Gambl Stud 1996; 12: 471-3.

5. American Psychiatric Association. Diagnostic and statistical manual of mental disorders. 5th ed. Arlington, American Psychiatric Publishing. 2013.

6. Lin YH, Chang LR, Lee YH, Tseng HW, Kuo TB, Chen SH. Development and validation of the Smartphone Addiction Inventory (SPAI). PLoS One 2014; 9: e98312.

7. Foerster M, Roser K, Schoeni A, Röösli M. Problematic mobile phone use in adolescents: derivation of a short scale MPPUS-10. Int J Public Health 2015; 60: 277-86.

8. Cha SS, Seo BK. Smartphone use and smartphone addiction in middle school students in Korea: prevalence, social networking service, and game use. Health Psychol Open 2018; 5: 2055102918755046.

9. Haug S, Castro RP, Kwon M, Filler A, Kowatsch T, Schaub MP. Smartphone use and smartphone addiction among young people in Switzerland. J Behav Addict 2015; 4: 299-307.

10. Lee J, Seo K. The comparison of cervical repositioning errors according to smartphone addiction grades. J Phys Ther Sci 2014; 26: 595-8.

11. Mendonça CR, Noll M, de Carvalho Santos ASEA, Rodrigues APDS, Silveira EA. High prevalence of musculoskeletal pain in individuals with severe obesity: sites, intensity, and associated factors. Korean J Pain 2020; 33: 245-57.

12. Zirek E, Mustafaoglu R, Yasaci Z, Griffiths MD. A systematic review of musculoskeletal complaints, symptoms, and pathologies related to mobile phone usage. Musculoskelet Sci Pract 2020; 49: 102196.

13. Berolo S, Wells RP, Amick BC 3rd. Musculoskeletal symptoms among mobile hand-held device users and their relationship to device use: a preliminary study in a Canadian university population. Appl Ergon 2011; 42: 371-8.

14. AlAbdulwahab SS, Kachanathu SJ, AlMotairi MS. Smartphone use addiction can cause neck disability. Musculoskeletal Care 2017; 15: 10-2.

15. Lau KT, Cheung KY, Chan KB, Chan MH, Lo KY, Chiu TT. Relationships between sagittal postures of thoracic and cervical spine, presence of neck pain, neck pain severity and disability. Man Ther 2010; 15: 457-62.

16. Sharan D, Mohandoss M, Ranganathan R, Jose J. Musculoskeletal disorders of the upper extremities due to extensive usage of hand held devices. Ann Occup Environ Med 2014; 26: 22 .

17. Kim HJ, Kim JS. The relationship between smartphone use and subjective musculoskeletal symptoms and university students. J Phys Ther Sci 2015; 27: 575-9.

18. Queiroz LB, Lourenço B, Silva LEV, Lourenço DMR, Silva CA. Musculoskeletal pain and musculoskeletal syndromes in adolescents are related to electronic devices. J Pediatr (Rio J) 2018; 94: 673-9.

19. Alruzayhi MK, Almuhaini MS, Alwassel AI, Alateeq OM. The effect of smartphone usage on the upper extremity performance among Saudi youth, KSA. Rom J Rhinol 2018; 8: 47-53.

20. Samaha M, Hawi NS. Relationships among smartphone addiction, stress, academic performance, and satisfaction with life. Comput Human Behav 2016; 57: 321-5.

21. Matar Boumosleh J, Jaalouk D. Depression, anxiety, and smartphone addiction in university students- a cross sectional study. PLoS One 2017; 12: e0182239.

22. Bian M, Leung L. Linking loneliness, shyness, smartphone addiction symptoms, and patterns of smartphone use to social capital. Soc Sci Comput Rev 2015; 33: 61-79.

23. Gustafsson E, Thomée S, Grimby-Ekman A, Hagberg M. Texting on mobile phones and musculoskeletal disorders in young adults: a five-year cohort study. Appl Ergon 2017; 58: 208-14.

24. Young KS. Internet addiction: the emergence of a new clinical disorder. Cyberpsychol Behav 1998; 1: 237-44.

25. Demirci K, Orhan H, Demirdas A, Akpinar A, Sert H. Validity and reliability of the Turkish version of the Smartphone Addiction Scale in a younger population. Klinik Psikofarmakol Bülteni 2014; 24: 226-34.

26. Yang SY, Chen MD, Huang YC, Lin CY, Chang JH. Association between smartphone use and musculoskeletal discomfort in adolescent students. J Community Health 2017; 42: 423-30.

27. Kahraman T, Genç A, Göz E. The Nordic Musculoskeletal Questionnaire: cross-cultural adaptation into Turkish assessing its psychometric properties. Disabil Rehabil 2016; 38: 2153-60.

28. Demirci K, Akgönül M, Akpinar A. Relationship of smartphone use severity with sleep quality, depression, and anxiety in university students. J Behav Addict 2015; 4: 85-92.

29. Hegazy AA, Alkhail BA, Awadalla NJ, Qadi M, Al-Ahmadi J. 
Mobile phone use and risk of adverse health impacts among medical students in Jeddah, Saudi Arabia. Br J Med Med Res 2016; 15: 1-11.

30. Bonney RA, Corlett EN. Head posture and loading of the cervical spine. Appl Ergon 2002; 33: 415-7.

31. Kim SY, Koo SJ. Effect of duration of smartphone use on muscle fatigue and pain caused by forward head posture in adults. J Phys Ther Sci 2016; 28: 1669-72.

32. Hakala PT, Rimpelä AH, Saarni LA, Salminen JJ. Frequent computer-related activities increase the risk of neck-shoulder and low back pain in adolescents. Eur J Public Health
2006; 16: 536-41.

33. Toh SH, Coenen P, Howie EK, Mukherjee S, Mackey DA, Straker LM. Mobile touch screen device use and associations with musculoskeletal symptoms and visual health in a nationally representative sample of Singaporean adolescents. Ergonomics 2019; 62: 778-93.

34. Chen B, Liu F, Ding S, Ying X, Wang L, Wen Y. Gender differences in factors associated with smartphone addiction: a cross-sectional study among medical college students. BMC Psychiatry 2017; 17: 341. 